\title{
Wood Species Recognition System based on Improved Basic Grey Level Aura Matrix as feature extractor
}

\author{
Mohd Iz'aan Paiz Zamri ${ }^{1}$, Anis Salwa Mohd Khairuddin, Norrima Mokhtar ${ }^{1}$, Rubiyah Yusof ${ }^{2}$ \\ ${ }^{I}$ Department of Electrical Engineering, Faculty of Engineering, University of Malaya, 50603 Kuala Lumpur, Malaysia \\ ${ }^{2}$ Malaysia Japan International Institute of Technology, Universiti Teknologi Malaysia, Kuala Lumpur Malaysia \\ E-mails: izanpaiz@gmail.com,anissalwa@um.edu.my
}

www.um.edu.my, www.fk.um.edu.my,www.mjiit.utm.my

\begin{abstract}
An automated wood species recognition system is designed to perform wood inspection at custom checkpoints in order to avoid illegal logging. The system that includes image acquisition, feature extraction and classification is able to classify the 52 wood species. There are 100 images taken from the each wood species is then divided into training and testing samples for classification. In order to differentiate the wood species precisely, an effective feature extractor is necessary to extract the most distinguished features from the wood surface. In this research, an Improved Basic Grey Level Aura Matrix (I-BGLAM) technique is proposed to extract 136 features from the wood image. The technique has smaller feature dimension and is rotational invariant due to the considered significant feature extract from the wood image. Support vector machine (SVM) is used to classify the wood species. The proposed system shows good classification accuracy compared to previous works.
\end{abstract}

Keywords: image classification, wood texture, wood species, support vector machine, pattern recognition.

\section{Introduction}

Traditional wood identification system which is based solely on human expertise is not practical nowadays. Several automatic wood species recognition have been developed based on spectral analysis and image analysis. The wood species identification based on the spectrum analysis involves a lot of time, money and specialists as shown in the previous research ${ }^{1-6}$. In addition, the technique is not suitable to be applied in real life applications by non-experts and is more practical to do it in the laboratory rather than in the field. On the other hand, the image-based processing provides a simpler method in classifying the wood species. The method involves only the inspection of a cross section of the wood surface that can be determined by human naked eye with the aid of magnifying lens. Refs. 7 and 8 develop an automatic tropical wood species recognition system to classify 20 wood species and 10 wood species respectively. In both works, Grey Level Co-occurrence Matrix (GLCM) is used as the feature extractor to extract the features from the wood texture. After the database is increased to 52 wood species the accuracy dropped to $50 \%$ indicating that the GLCM in the previous work is inadequate for large wood database. This is due to the unvaried nature of macroscopic anatomy image of the wood, but it contains 
a visual texture - variation of intensities which form certain repeated arrangements ${ }^{9}$.

In order to improve the performance of the wood species recognition system, a reliable and precise feature extractor is needed. To correctly extract the wood features from the wood texture and overcome the limitations of GLCM, a new feature extractor, Improved Basic Grey Level Aura Matrix (I-BGLAM) is proposed. Support Vector Machine (SVM) is used as classifier to classify the wood species based on the feature extracted from the I-BGLAM. Several phases which consist of image acquisition, feature extraction and classification are proposed as the design of the automated wood recognition system.

\section{Proposed Methodologies}

The proposed wood identification system based on image analysis consists of several parts: image acquisition, feature extraction and classification. The illustration of the proposed system is shown in the Fig. 1.

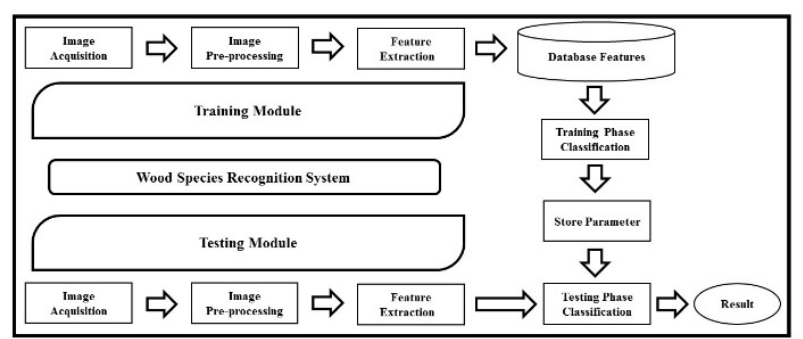

Fig. 1. Proposed wood identification system

\subsection{Image acquisition}

In image acquisition stage, images of wood surface are captured by a specially designed portable camera. The portable camera has 10 times magnification, which is the same specification as the magnifier used in manual wood identification process. The size of each image is $768 \times 576$ pixels.

The wood images are pre-processed using homomorphic filters to enhance the image presentation. Homomorphic filtering uses a linear filter to do a nonlinear mapping to a different domain and later it is mapped back to the original domain. This algorithm reduces all unwanted illumination and reflectance on the image. Image brightness is also normalized, thus enhancing the contrast of the image. The algorithm of homomorphic filtering is explained in more detail in Ref. 10.

\subsection{I-BGLAM feature extractor}

In order to overcome the limitations of the conventional BGLAM technique, an improved BGLAM feature extractor is proposed. Basically, the improved BGLAM feature extractor is rotational invariant and the size of feature dimension is reduced by considering only the discriminative features. Besides that, I-BGLAM features are directly calculated from the grey level mapping of images which overcome the limitations of GLCM.

This is done by creating a new characteristic vector which combine of the occurrence of 00 together, the occurrences of 01 and 10 together and the occurrences of 11 together.

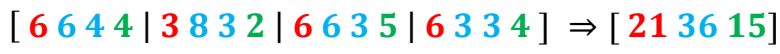

For example,

$6+3+6+6=21,4+2+5+4=15$ and

$6+4+8+3+6+3+3+3=3$

This is how a 32 feature vector BGLAM becomes a 3 feature vector invariant to image rotation. The number of I-BGLAM features depends only on the grey level segmentation as shown in Eq. (1):

$$
N_{f}=\frac{G L \times(G L+1)}{2}
$$

Where $N_{f}$ is the number of features and $G L$ represents grey level.

Consequently, the number of features becomes less at the same time. In this research, the numbers of grey level experimented is $2,4,8,16$. Finally, a grey level of 16, which gives 136 features is chosen for the final experiment because it gives the highest classification accuracy compared to other grey levels.

\subsection{Classification}

The classification process is performed by training a classifier model that works by differentiating features of the class of observed collected data. Support Vector Machine (SVM) is a useful tool as a learning algorithm in analyzing and recognizing data. Previous works in 
Refs. 11-17 shows that the implementation of SVM classifier result to high classification rate.

\section{Results and Discussion}

The experiment was conducted by using 90 training samples and 10 test samples for each wood species and is repeated for 30 iterations. From the result obtained (Table 1), the classification accuracy for training samples for all set showed $100 \%$ in accuracy. The computed training accuracy showed that SVM is able to train the training model and identify the trained data in training samples database accurately. The classifier managed to classify the test samples with an average accuracy

$99.84 \pm 0.23 \%$ for 52 wood species.

Table 1. Average classification accuracy

\begin{tabular}{|c|c|}
\hline Set & $\begin{array}{c}\text { Classification } \\
\text { accuracy } \mathbf{( \% )}\end{array}$ \\
\hline Train & 100 \\
\hline Test & $99.84 \pm 0.23$ \\
\hline
\end{tabular}

The proposed system showed an increment in test accuracy compared to the previous works done shown in the Table 2.

Table 2. Previous works on wood species recognition system based on image texture

\begin{tabular}{|c|c|l|c|}
\hline Ref. & $\begin{array}{c}\text { No of species } \\
\text { (No of } \\
\text { samples) }\end{array}$ & $\begin{array}{l}\text { Texture feature } \\
\text { (No of features) }\end{array}$ & $\begin{array}{c}\text { Classification } \\
\text { accuracy (\%) }\end{array}$ \\
\hline 7 & $20(1949)$ & GLCM (20) & 95.00 \\
\hline 17 & $7(101)$ & $\begin{array}{l}\text { Image } \\
\text { segmentation (5) }\end{array}$ & 93.00 \\
\hline 18 & $5(250)$ & GLCM (20) & 72.00 \\
\hline 19 & $11(347)$ & $\begin{array}{l}\text { GLCM (24), } \\
\text { Color (18) }\end{array}$ & 82.00 \\
\hline 20 & $22(1270)$ & $\begin{array}{l}\text { GLCM (24), } \\
\text { Color (18) }\end{array}$ & 80.80 \\
\hline 21 & $25(1250)$ & GLCM (124) & 68.40 \\
\hline 22 & $10(1000)$ & GLCM (3) & 95.00 \\
\hline 23 & $25(500)$ & GLCM (44) & 92.60 \\
\hline 24 & $20(2010)$ & $\begin{array}{l}\text { Gabor filters- } \\
\text { GLCM (200) }\end{array}$ & 91.00 \\
\hline 25 & $30(3000)$ & $\begin{array}{l}\text { GLCM (200), } \\
\text { Gabor filters } \\
\text { (80),GLCM(20) }\end{array}$ & 90.33 \\
\hline
\end{tabular}

\section{Conclusion}

The classification of features extracted from I-BGLAM using SVM classifier shows an improvement in the classification accuracy compared to previous proposed systems. The features extracted from the wood species using I-BGLAM as feature extractor are more distinct, thus improved the accuracy of the wood recognition system. In future, an advance wood recognition system is needed to overcome the size of wood database and able to differentiate the wood species that contain more likely features with each other. A robust feature extractor that capable to extract the most discriminant features from the wood texture is the aim for development in future.

\section{Acknowledgements}

The authors would like to thank Malaysian Ministry of Higher Education (MOHE) and University of Malaya for funding this research through High Impact Research Grant (UM.C/HIR/MOHE/ENG/16) and BKP Grant (BK047-204). The authors also would like to thank Forest Research Institute of Malaysia (FRIM) for providing us with the wood samples. The first author would like to thank to Ministry of Higher Education (MOHE) for awarded him My Master scholarship.

\section{References}

1. Fuentealba, C., et al. Wood products identification by internal characteristics readings. in Industrial Technology, 2004. IEEE ICIT'04. 2004 IEEE International Conference on. 2004. IEEE.

2. Choffel, D. Automation of wood mechanical grading: coupling of vision and microwave devices. in Photonics East'99. 1999. International Society for Optics and Photonics.

3. Piuri, V. and F. Scotti, Design of an automatic wood types classification system by using fluorescence spectra. Systems, Man, and Cybernetics, Part C: Applications and Reviews, IEEE Transactions on, 2010. 40(3): p. 358-366.

4. Baas, P., E. Wheeler, and M. Chase, Dicotyledonous wood anatomy and the APG system of angiosperm classification. Botanical Journal of the Linnean Society, 2000. 134(1-2): p. 3-17.

5. Rojas, J., et al., Wood species identification using stresswave analysis in the audible range. Applied Acoustics, 2011. 72(12): p. 934-942. 
6. Puttonen, E., et al., Tree species classification from fused active hyperspectral reflectance and LIDAR measurements. Forest Ecology and Management, 2010. 260(10): p. 1843-1852.

7. Khalid, M., et al., Design of an intelligent wood species recognition system. International Journal of Simulation System, Science and Technology, 2008. 9(3): p. 9-19.

8. Bremananth, R., B. Nithya, and R. Saipriya. Wood species recognition using GLCM and correlation. in Advances in Recent Technologies in Communication and Computing, 2009. ARTCom'09. International Conference on. 2009. IEEE.

9. Petrou, M. and P.G. Sevilla, Image Processing. Texture: Dealing with Texture. 2006, Wiley John and Sons.

10. Gonzalez, R.C. and R.E. Woods, Digital image processing 3rd edition. 2007, Prentice Hall.

11. Martins, J., et al., A database for automatic classification of forest species. Machine Vision and Applications, 2013. 24(3): p. 567-578.

12. Martins, J., et al., Forest species recognition based on dynamic classifier selection and dissimilarity feature vector representation. Machine Vision and Applications, 2015. 26(2-3): p. 279-293.

13. Paula Filho, P.L., et al., Forest species recognition using macroscopic images. Machine Vision and Applications, 2014. 25(4): p. 1019-1031.

14. Yadav, A.R., et al., Multiresolution local binary pattern variants based texture feature extraction techniques for efficient classification of microscopic images of hardwood species. Applied Soft Computing, 2015. 32: p. 101-112.

15. $\mathrm{Hj}, \mathrm{W}$., et al., Wood recognition using image texture features. PloS one, 2013. 8(10): p. e76101.

16. Turhan, K. and B. Serdar, Support vector machines in wood identification: the case of three Salix species from Turkey. Turk J Agric For, 2013. 37: p. 249-256.
17. Mallik, A., et al., Classification of wood micrographs by image segmentation. Chemometrics and intelligent laboratory systems, 2011. 107(2): p. 351-362.

18. Tou, J.Y., P.Y. Lau, and Y.H. Tay. Computer vision-based wood recognition system. in Proceedings of International Workshop on Advanced Image Technology. 2007. Citeseer.

19. de Paula Filho, P.L., L.S. Oliveira, and A.S. Britto Jr. $A$ database for forest species recognition. in Proceedings of the XXII Brazilian Symposium on Computer Graphics and Image Processing. 2009.

20. Filho, P.L.P., et al. Forest Species Recognition Using Color-Based Features. in Pattern Recognition (ICPR), 2010 20th International Conference on. 2010.

21. Hasan, A.F., et al., Application of Binary Particle Swarm Optimization in Automatic Classification of Wood Species using Gray Level Co-Occurence Matrix and K-Nearest Neighbor. Int. J. Sci. Eng. Res, 2013. 4: p. 50-55.

22. Mohan, S., K. Venkatachalapathy, and P. Sudhakar, An Intelligent Recognition System For Identification Of Wood Species. Journal of Computer Science, 2014. 10(7): p. 1231-1237.

23. Yadav, A.R., et al. Classification of hardwood species using ann classifier. in Computer Vision, Pattern Recognition, Image Processing and Graphics (NCVPRIPG), 2013 Fourth National Conference on. 2013. IEEE.

24. Yusof, R., N.R. Rosli, and M. Khalid. Tropical wood species recognition based on Gabor filter. in Image and Signal Processing, 2009. CISP'09. 2nd International Congress on. 2009. IEEE.

25. Yusof, R., N.R. Rosli, and M. Khalid. Using gabor filters as image multiplier for tropical wood species recognition system. in Computer Modelling and Simulation (UKSim), 2010 12th International Conference on. 2010. IEEE. 